voit que l'ensemble $M_{2}=R-M_{1}$ est alors dense dans $R$. 2) Chaque point de $M_{1}$ est point limite de $M_{1}$ dú côté gauche et du côté droit ${ }^{7}$ ). Posons ensuite: $f(x, y)=0$ pour $x \in M_{2} ; f(x, y)=-1$ pour $x \in M_{1}$, $x-y \in M_{1} ; f(x, y)=-1$ pour $x \in M_{1}, x-y \in M_{2}$. Désignons par 8 la droite $y=0$ et par $d_{1}, d_{2}, d_{3}$ les directions des demidroites suivantes: $y>0, x=0 ; y>0, y=x ; y>0, y=-x$. Soit $x \in M_{1}, P=(x ; 0)$; on voit aisément que chacun des trois ensembles $E\left(P, d_{1}\right), E\left(P, d_{2}\right)$ $E\left(P, d_{3}\right)$ est composé de deux nombres, à savoir de $-1,1$, de 0,1 et de $0,-1$ respectivement. Donc la relation (7) est en défaut pour chaque point $P$ d'un ensemble non dénombrable (à savoir pour $\left.P=(x, 0), x \in M_{1}\right)$.

Remarque II. Pour donner une application de notre théorème, soit $F(x)$ une fonction réelle et finie d'une variable réelle. Posons

$$
f(x, x)=0, \quad f(x, y)=\frac{F(y)-F(x)}{y-x} \quad \text { pour } \quad y \neq x .
$$

Soit $s$ la droite $y=x$; soient $d_{1}$ et $d_{2}$ les directions des demidroites $y>0, x=0$ et $x<0, y=0$ respectivement. Pour $P=(x, x)$, les valeurs de $f(\xi, \eta)$ sur les demidroites $\overrightarrow{P d}_{1}$ et $\overrightarrow{P d}_{2}$ sont données respectivement par $\frac{1}{h}(F(x+h)-F(x))$ et par $\frac{1}{h}(F(x)-F(x-h))$ (pour $\left.h>0\right)$. Donc: il existe un ensemble dénombrable $D$ tel que l'on peut, à chaque $\left.x \in R-D^{8}\right)$, faire correspondre deux suites $h_{1}, h_{2}, \ldots ; k_{1}, k_{2}, \ldots$ telles que

$h_{n}>0, h_{n} \rightarrow 0 ; \quad k_{n}<0, k_{n} \rightarrow 0 ; \lim _{n=\infty} \frac{F\left(x+h_{n}\right)-F(x)}{h_{n}}=\lim _{n=\infty} \frac{F\left(x+k_{n}\right)-F(x)}{k_{n}}$.

C'est un théorème un peu plus précis que le théorème bien connu d'après lequel on a pour $x \in R-D$ :

$$
\limsup _{h=0+} \frac{F(x+h)-F(x)}{h} \geqq \lim _{h=0-} \frac{F(x+h)-F(x)}{h} .
$$

7) On peut prendre pour $M_{1}$ p.ex. l'ensemble de tous les nombres irration. nels de la forme $\sum_{n=1}^{\infty} a_{n} \cdot 3^{-n}$, chaque $a_{n}$ étant égal à 0 ou à 2.

8) $R$ désigne l'ensemble de tous les nombres réels.

\section{Sur un théorème de M. Roger.}

Par

\section{S. S a ks (Warszawa).}

Dans ma Note „Sur quelques proprietés métriques d'ensembles" (Fund. Math., 26 (1936), pp. 234-240) contenant une généralisation des théorèmes bien connus de M. Denjoy sur les nombres dérivés, j'ai observé que les méthodes employées par moi pour le cas du plan peuvent être appliquées, sans modification essentielle, pour obtenir les résultats analogues, concernant l'espace et signalés par M. F. Roger dans la série de ses communications de C. R. J'ai accompagné cette observation par des exemples de quelques-uns des théorèmes de M. Roger dans l'espace, les autres théorèmes m'ayant semblé être des conséquences assez proches de ceux que j'ai établis pour' le plan. Or, puisque M. Roger vient d'observer dans le renvoi de sa note récente de O. R. (202 (1936), pp. 1403-1405) que ma remarque "reste muette" sur les autres de ses résultats, qu'il considère comme moins simples et plus importants, j'en publie ici une démonstration détaillée, pour montrer explicitement qu'ils se réduisent aussitôt au cas du plan. Je les formule ici sous la forme du théorème suivant dans l'espace à 3 dimensions, l'extension sur $n \geqslant 3$ dimensions (de ce théorème, ainsi que des résultats mentionnés dans ma note précédente) s'obtenant facilement par induction.

$R$ désignant un ensemble dans l'espace à 3 dimensions, l'ensemble $P$ des points a de $R$ où $\operatorname{contg}_{R}$ a laisse échapper un plan entier est la somme d'une infinité au plus dénombrable d'ensembles de longueur finie, et dans tout point de $P$, excepté au plus un ensemble de longueur nulle, l'ensemble $R$ possède une tangente unique. 
Soit $\left\{\theta_{n}\right\}$ une suite de directions, partout dense dans l'espace. Désignons par $P_{n, m}$ l'ensemble des points $a$ de $P$ tels que $\left|\cos \left(a x, \theta_{n}\right)\right|>1 / m$ pour tout point $x$ de $R$ situé à une distance de $a$ moindre que $1 / m$. Décomposons chaque ensemble $P_{n, m}$ en une suite $\left\{P_{n, m, k\rangle_{k=1,2, \ldots}}\right.$ d'ensembles de diamètre moindre que $1 / m$. On a alors

$$
P=\sum_{n, m} P_{n, m}=\sum_{n, m, k} P_{n, m, k} .
$$

Fixons, pour l'instant, trois indices $n=n_{0}, m=m_{0}$ et $k=k_{0}$, et posons, pour abréger, $A=P_{n_{0}, m_{0}, k_{0}}$. Choisissons un nouveau système des coordonnées $\xi \eta \xi$, en prenant pour l'axe $O \zeta$ la droite de direction $\theta_{n_{i}}$. Nous désignerons généralement, par $Q_{\xi \zeta}$ et $Q_{\eta \zeta}$, lorsque $Q$ est un ensemble ou un point, les projections de $Q$ sur les plans $\xi \zeta$ et $\eta \zeta$ respectivement.

On a $|\cos (\overrightarrow{a x}, O \zeta)|>1 / m_{0}$ toutes les fois que $a \epsilon A, x \in R$ et $0<\varrho(a, x)<1 / m_{0}$. Il en résulte immédiatement que, dans le plan $\xi \zeta$, le contingent de l'ensemble $R_{\xi \zeta}$ laisse échapper la droite parallèle à l'axe des $\xi$ en tout point de l'ensemble $A_{\text {š. }}$. Parsuite, en vertu du

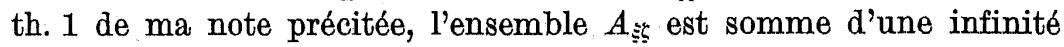
au plus dénombrable d'ensembles de longueur finie et $R_{55}$ possède une tangente unique dans tout point de $A_{\xi 5}$, excepté au plus un ensemble $M$ de longueur nulle. Pareillement, l'ensemble $R_{\eta 7}$ possède une tangente unique dans tout point de $A_{\eta 5}$, excepté au plus un ensemble $N$ de longueur nulle, Par conséquent, l'ensemble $R$ possède une tangente unique dans chaque point $a \operatorname{de} A$, excepté tout au plus le cas où $a_{5 \leq \epsilon} \in M$ ou bien $a_{\eta \eta} \epsilon N$. Or, on constate aisément que les deux quotients $\varrho(a, b) / \varrho\left(a_{\xi \zeta}, b_{\xi \zeta}\right)$ et $\varrho(a, b) / \varrho\left(a_{\eta \xi}, b_{\eta \xi}\right)$ restent bornés (inférieurs à $m_{0}$ ), quand $a$ et $b$ parcourent l'ensemble $A$. Parsuite l'ensemble des points exceptionnels de $A=P_{n_{0}, m_{0}, k_{0}}$ où $R$ ne possède pas de tangente unique est de longueur nulle, en même temps que les ensembles $M$ et $N$. Pour la même raison l'ensemble $A=P_{n_{0}, m_{0}, k_{0}}$ est somme d'une infinité au plus dénombrable d'ensembles de longueur finie, en même temps que sa projection $A_{\xi \xi t}$. Cela achève la démonstration en vertu de l'identité $(*)$.

\section{Sur les espaces multicohérents I.}

\author{
Par \\ Samuel Eilenberg (Warszawa).
}

Un continu localement connexe $X$ est dit unicohérent, lorsque'en le décomposant de n'importe quelle manière en deux continus:

$$
X=X_{1}+X_{2} \text {, }
$$

leur partie commune $X_{1} \cdot X_{2}$ est toujours connexe (donc un continu). Dans le cas contraire, $X$ est dit multicohérent. L'objet du présent ouvrage ${ }^{1}$ ) est d'étudier cette propriété au point de vue quantitatif. Posons dans ce but

$$
\left.r(X)=\sup \left[b_{0}\left(X_{1} \cdot X_{2}\right)-1\right]{ }^{2}\right),
$$

en faisant le couple des continus $X_{1}, X_{2}$ parcourir toutes les décompositions possibles de la forme (*).

L'égalité $r(X)=0$ exprime donc évidemment l'unicohérence (1-cohérence) de $X$. Plus généralement, nous appellerons $X$ n-cohérent, lorsque $r(X)+1=n$.

L'unicohérence est caractérisée, comme on sait ${ }^{3}$ ), par l'évanouissement du premier nombre de Betti. On pourrait donc croire que le nombre $r(X)$ se laisse également exprimer, et d'une manière simple, à l'aide du premier nombre de Betti. Or, il n'en est rien, et nous verrons que l'étude du nombre $r(X)$, et de certaines généralisations de ce nombre, permet de saisir des propriétés plus profondes que celles qui se traduisent par le premier nombre de Betti. Ajoutons que le "Produktsatz" pour le nombre $r(X)$ est tout à fait différent de celui pour le premier nombre de Betti:

1) Les résultats principaux exposés ici ont été présentés à la Société Polonaise de Mathématiques, Section de Varsovie, séances du 15. 9. 1935 et du 28. 2. 1936 .

2) $b_{0}(X)=$ nombre de composantes de $Y$, lorsque ce nombre est fini; dans le cas contraire $b_{0}(X)=\infty$.

3) K. Borsuk, Fund. Math. 20 (1933), p. 230; E. Čech, ibid., p. 232. 